\title{
Das Recht auf Nichtwissen im Kontext prädiktiver Gendiagnostik
}


Ariane Schroeder

\section{Das Recht auf \\ Nichtwissen im \\ Kontext prädiktiver \\ Gendiagnostik}

Eine Studie zum ethisch verantworteten Umgang mit den Grenzen des Wissens

Springer VS 
Ariane Schroeder

Augsburg, Deutschland

Dissertation an der Philosophisch-Sozialwissenschaftlichen Fakultät der Universität Augsburg, 2013

ISBN 978-3-658-07145-5

ISBN 978-3-658-07146-2 (eBook)

DOI $10.1007 / 978-3-658-07146-2$

Die Deutsche Nationalbibliothek verzeichnet diese Publikation in der Deutschen Nationalbibliografie; detaillierte bibliografische Daten sind im Internet über http://dnb.d-nb.de abrufbar.

\section{Springer VS}

(C) Springer Fachmedien Wiesbaden 2015

Das Werk einschließlich aller seiner Teile ist urheberrechtlich geschützt. Jede Verwertung, die nicht ausdrücklich vom Urheberrechtsgesetz zugelassen ist, bedarf der vorherigen $\mathrm{Zu}$ stimmung des Verlags. Das gilt insbesondere für Vervielfältigungen, Bearbeitungen, Übersetzungen, Mikroverfilmungen und die Einspeicherung und Verarbeitung in elektronischen Systemen.

Die Wiedergabe von Gebrauchsnamen, Handelsnamen, Warenbezeichnungen usw. in diesem Werk berechtigt auch ohne besondere Kennzeichnung nicht zu der Annahme, dass solche Namen im Sinne der Warenzeichen- und Markenschutz-Gesetzgebung als frei zu betrachten wären und daher von jedermann benutzt werden dürften.

Gedruckt auf säurefreiem und chlorfrei gebleichtem Papier

Springer VS ist eine Marke von Springer DE. Springer DE ist Teil der Fachverlagsgruppe Springer Science+Business Media.

www.springer-vs.de 


\section{Vorwort}

„Wo ist die Grenze zwischen einem Fortschritt, der dem Menschen dient, und einem Fortschritt, der in eine prinzipielle Selbstüberforderung des Menschen mündet, weil ein Wissen erzeugt wird, dessen verantwortliche Handhabung er nicht mehr gewährleisten und gesellschaftlich sicherstellen kann?"1

Ein solches grenzwertiges Wissen lässt sich mithilfe der Prädiktiven Gendiagnostik erheben. Grenzwertig ist das Wissen, weil es einerseits die Identifikation individueller genetischer Krankheitsdispositionen ermöglicht und auf dieser Grundlage den Zugang zu bestimmten präventiv-medizinischen Strategien. Diese zielen auf die Verhinderung einer möglichen Krankheitsmanifestation, auf die zeitliche Verzögerung des Erkrankungseintritts sowie auf die positive Beeinflussung des Krankheitsverlaufs. Andererseits kann das Wissen um eine in der $\mathrm{Zu}$ kunft liegende schwere Erkrankung eine Bürde darstellen. So ist das ermittelbare prädiktive Wissen zumeist probabilistischer Natur. Der Betreffende ${ }^{2}$ sieht sich zwar mit einem individuellen Erkrankungsrisiko konfrontiert, aber ob, wann und mit welchem Verlauf die drohende Krankheit tatsächlich in Erscheinung tritt, ist zumeist nicht vorhersehbar. Prädiktiv-genetisches Wissen kann überfordern, Lebensziele und -pläne radikal infrage stellen und den Menschen in eine Situation empfundener Ausweglosigkeit entlassen. Der Einzelne als Adressat des prädiktiv-gendiagnostischen Testangebots ist, vor dem Hintergrund der bereits in weiten Teilen vollzogenen Umdeutung der Erkrankungsgefahr in das -risiko, zu einer selbstverantworteten Stellungnahme aufgefordert. Über die individualethi-

\footnotetext{
$1 \quad$ M. Vogt, Was wird aus meiner Entscheidung? Folgenabschätzung unter komplexen Bedingungen, in: R. M. Scheule, Ethik der Entscheidung. Entscheidungshilfen im interdisziplinären Diskurs, Regensburg 2009, 47-75, hier: 66.

2 Die Verwendung der maskulinen Form dient ausschließlich der besseren Lesbarkeit.
} 
sche Perspektive hinaus scheint das gendiagnostische Wissen ebenso in gesamtgesellschaftlicher Hinsicht bedeutsam zu sein, stellt es doch, integriert in eine patientenorientierte Medizin auf der Basis von Prädiktion und Prävention, längerfristig eine finanzielle Entlastung des Gesundheitswesens in Aussicht. Die Frage der Erhebung und Nutzung prädiktiven Wissens enthält damit eine Dimension, aus deren Optik der erklärte Wissensverzicht begründungsbedürftig, wenn nicht sogar kontextbezogen verantwortungslos und inakzeptabel erscheint. Angesichts der psychosozialen Ambivalenzen des prädiktiv-genetischen Wissens und um einer möglichen Überforderung des Einzelnen durch ein unter Umständen nur eingeschränkt nutzbares Wissen entgegenzuwirken, findet das Recht auf Nichtwissen innerhalb des im Februar 2010 in Kraft getretenen Gendiagnostikgesetzes (GenDG) ausdrücklich Berücksichtigung. Primär bezeichnet der auf die allgemeinen Persönlichkeitsrechte gegründete Anspruch ein defensives Schutzrecht vor wissensverursachten Lebensqualitätseinbußen und sozialen Sanktionen.

Dezidiert nimmt die angestrebte ethische Auseinandersetzung unter theologisch-ethischer Perspektive die einwilligungsfähige Person als Entscheidungsund Verantwortungsträger in den Blick ${ }^{3}$. Es ist zu hinterfragen, ob der erklärte Wissensverzicht nicht in erster Linie eine zutiefst personale und freiheitseröffnende Option im Umgang mit den Wissensangeboten der modernen Biomedizin darstellt. Das Recht auf Nichtwissen würde dann nicht schwerpunktmäßig als passives Schutzrecht begriffen werden. Vielmehr wäre es Ausdruck der aktiven Lebensgestaltung des unter dem sittlichen Anspruch stehenden Subjekts. Eine solche Interpretation, ließe sie sich argumentativ begründen, würde den Eigenwert des Rechts auf Nichtwissen gegenüber dessen Unterordnung unter das festetablierte Prinzip des Informed Consents unterstreichen. $\mathrm{Zu}$ diesem träte der erklärte Wissensverzicht in notwendige Ergänzung. Der Versuch einer fallbezogenen positiven Legitimierung des Rechts auf Nichtwissen auf Basis einer Verteidigung des autonomen Subjekts bildet das eine Kernanliegen der ethischen Reflexion. Dabei, das ist bereits an dieser Stelle zu betonen, darf das Recht auf

Nicht behandelt werden ethische Fragestellungen zur Präimplantationsdiagnostik, zur Pränataldiagnostik sowie zu genetischen Untersuchungen im Kindes- und Jugendalter. Auch genetische Reihenuntersuchungen, Analysen zu Forschungszwecken sowie datenschutzrechtliche Überlegungen bleiben unberücksichtigt. 
Nichtwissen nicht im Sinne eines pauschalen Abwehrrechts missverstanden werden. Kritisch sind mögliche Grenzen auszuloten. Autonomie unterliegt stets limitierenden Faktoren. Die ethische Reflexion hat daher der begrenzten menschlichen Einsichts- und Handlungsfähigkeit Rechnung zu tragen. Im Blick auf die Prädiktive Gendiagnostik erschweren zudem wissenschaftsimmanente Unsicherheiten sowie ein eklatanter Mangel an ethischen Orientierungshilfen die persönliche Entscheidungsfindung. Dem letztgenannten Defizit soll begegnet werden. Als eine Weise ethischen Nachdenkens steht die theologische Ethik im Dienste der Lebensdeutung sowie der Lebensbewältigung. Aus einem subjektzentrierten und für hermeneutische Fragen sensibilisierten Blickwinkel sind jenseits einseitig zweckrationaler Erwägungen personale Ressourcen zur Entwicklung einer begründeten, tragfähigen und moralisch vertretbaren Handlungsorientierung zu entdecken. In operationalisierbare Entscheidungshilfen überführt, sollen sie, unter deutlicher Berücksichtigung des naturwissenschaftlich-empirischen Kenntnisstands, auf kritisch-konstruktivem Wege Entscheidung herbeiführen und Entschiedenheit stabilisieren. Damit ist das zweite Kernanliegen der ethischen Überlegungen skizziert. 


\section{Danksagung}

Bei der vorliegenden Studie handelt es sich um eine gekürzte Fassung der Dissertationsschrift „Prädiktive Gendiagnostik und theologische Ethik des Nichtwissens. Eine aktuelle Bedarfsanzeige“. Diese entstand im Rahmen des von der Deutschen Forschungsgemeinschaft (DFG) geförderten Projektes „Ethik des Nichtwissens - Ein theologisch-ethisches Angebot" unter der Leitung von Prof. Dr. Klaus Arntz. Im Sommersemester 2013 wurde die Studie von der Philosophisch-Sozialwissenschaftlichen Fakultät der Universität Augsburg als Dissertation angenommen.

Herzlich möchte ich Prof. Dr. Klaus Arntz danken. Als Betreuer der Arbeit stand er mir in den vergangenen Jahren stets engagiert und kompetent zur Seite. Wertvolle Anregungen zur ethischen Auseinandersetzung mit den Wissens- und Nichtwissensbeständen in der modernen Biomedizin erhielt ich auch von meinen ehemaligen Kolleginnen und Kollegen. Ihnen sei für die konstruktive Zusammenarbeit gedankt. Ein herzlicher Dank gebührt Prof. Dr. Thomas Schärtl für die Erstellung des Zweitgutachtens.

Ganz besonders danke ich meiner Familie und meinen Freunden. Auf ihre Weise haben sie zum Gelingen dieser Arbeit beigetragen.

Kelheim, am 20. Juli 2014

Ariane Schroeder 


\section{Inhaltsverzeichnis}

$1 \quad$ Einführung ............................................................................................................. 1

2 Nichtwissen: ein anthropologisches Grundphänomen ............................. 9

2.1 Streben nach Sicherheit: ein allgemein menschliches

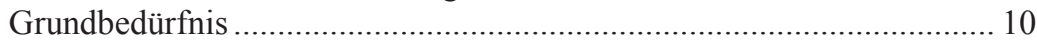

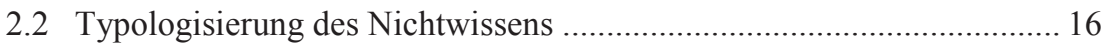

3 Risiko: eine moderne Bewältigungsstrategie des Nichtwissens ............ 27

3.1 Begriffsbestimmung unter soziologischer Perspektive .......................... 28

3.1.1 Risiko und Verantwortung ........................................................... 35

3.1.2 Entgrenzungstendenzen gegenwärtiger Zurechnungspraktiken ...... 37

3.2 „Embodied Risks“: ungesichertes Wissen in der modernen Medizin ..... 39

3.2.1 Tätigkeitsfelder genetischer Diagnostik ....................................... 42

3.2.2 Prädiktive Gendiagnostik am Beispiel der erblichen Brustkrebsvariante .................................................................. 45

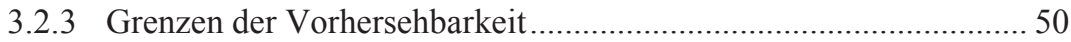

3.3 „Embodied Risks“ zwischen Empirie und sozialer Konstruktion........... 53

3.3.1 Risikogenese - nur eine Frage der richtigen Rhetorik? .................. 54

3.3.2 Vermittlung wissenschaftlichen Wissens als Herausforderung

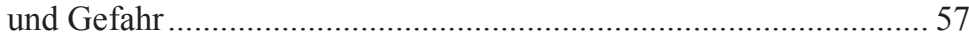

3.3.3 Die genetische Beratung: institutionalisiertes Gespräch und

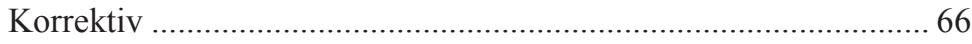

3.4 Prädiktives Wissen im Kontext des Verantwortungsbegriffs ................ 72

3.4.1 Tendenzen einer Genetifizierung der Gesellschaft? ....................... 73

3.4.2 Eigenverantwortung: eine gesundheitspolitische Schlüsselkategorie .................................................................. 83

3.5 Prädiktives Wissen zwischen Anspruch und Zumutung ......................... 96

3.5.1 Ambivalenzen des Wissens aus medizinischer Sicht ..................... 96

3.5.2 Ambivalenzen des Wissens aus psychologischer Sicht ................. 104 
3.5.3 Ambivalenzen des Wissens aus soziologischer Sicht.................... 114

3.6 Ertrag: Grenzen einer gesundheitsbezogenen Eigenverantwortung ...... 126

4 Das Recht auf Nichtwissen: ein technikaverser Irrläufer? .................. 131

4.1 Rechtliche Grundlegung ................................................................. 132

4.2 Autonomie in medizinischer Hinsicht................................................ 134

4.2.1 Das Modell der Patientenmündigkeit als Korrektiv ..................... 138

4.2.2 Der Informed Consent - Garant autonomer Entscheidung? .......... 141

4.2.3 Patientenautonomie im Rahmen der genetischen Beratung........... 146

4.2.4 Das Recht auf Nichtwissen im Kontext des Informed Consents ... 151

4.3 Das Recht auf Nichtwissen: ein partielles Abwehrrecht ...................... 154

4.3.1 Reichweitenbegrenzung unter prinzipienethischer Prämisse......... 156

4.3.2 Das Recht auf Nichtwissen im innerfamiliären

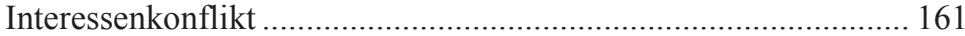

4.3.3 Das Recht auf Nichtwissen im wirtschaftlichen

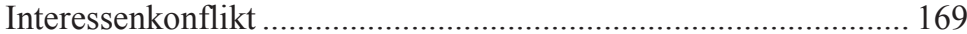

4.4 Ertrag: Das Recht auf Nichtwissen - eine freiheitseröffnende Option . 177

5 Prädiktives Wissen: die verdrängte Dimension der Leiblichkeit ....... 181

5.1 Leibvergessenheit in der modernen Medizin ....................................... 183

5.2 Leibsein und Leibhaben: Impulse aus leibphilosophischer Sicht ......... 187

5.2.1 Die Bedeutung der betroffenen Selbstgegebenheit....................... 187

5.2.2 Implikationen im Kontext der modernen Biomedizin ................... 192

5.3 Ertrag: Plädoyer für eine Rückbesinnung auf die Leiblichkeit ............. 196

6 Prädiktives Wissen aus hermeneutischer Sicht ................................... 199

6.1 Theologische Ethik als handlungsleitende Sinnwissenschaft .............. 201

6.2 Sinndeutung und gelebte Überzeugung: Quellen ethischen Urteilens .. 205

6.2.1 Die Bedeutung der Lebensgeschichte............................................ 206

6.2.2 Wissen aus Erfahrung: das Kriterium der Experienz..................... 210 
6.2.3 Bewährtes Lebenswissen: Ressource im Umgang mit Nichtwissen

6.3 Die Relevanz gelebter Überzeugungen in normativer Hinsicht............ 225

6.3.1 Autonomie unter anthropologisch-hermeneutischer Prämisse ...... 226

6.3.2 Autonomie im Horizont von Relationalität und endlicher Einsicht

6.3.3 Kritische Begleitung: ein zentraler Auftrag einer gen-ethischen Beratung

6.3.4 Das Recht auf Nichtwissen: Die Frage der faktischen sozialen Gültigkeit

6.4 Ertrag: Nichtwissen - ein Recht in Sinneinsicht und Verantwortung... 255

7 Theologische Hermeneutik der Lebensdeutung: ein Angebot ............. 263

7.1 Wissenschaftstheoretische Anmerkungen............................................ 264

7.2 Prädiktives Wissen aus dem Blickwinkel einer theologischen Ethik.... 267

7.2.1 Gesundheitsvorsorge und autonome Lebensgestaltung ................. 268

7.2.2 Grenzen einer ethisch vertretbaren Gesundheitsvorsorge.............. 271

7.2.3 Impulse für einen verantwortungsbewussten Einsatz prädiktiven

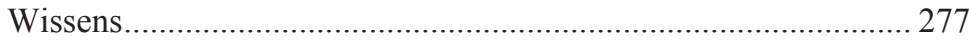

7.3 Ertrag: Gewahrwerdung der Endlichkeit - ein ethisches Desiderat...... 283

7.4 Ausblick: Mut zur gemäßigten Prävention ........................................ 288

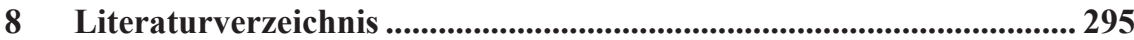

